\title{
Study on ERP II Decisions under Asymmetric Information: an Agency Approach
}

\author{
Wang Lina \\ School of Economics Management, \\ Liaoning University of Technology, Jinzhou, 121001, \\ Liaoning Province, P.R.China; \\ Room 3113,EHSAL Research Center of HUB, Stormstraat 1, \\ 1000 Brussels, Belgium
}

Lina1976113@126.com

\begin{abstract}
In this paper, the optimization investment policy decision of an ERP II implementation has been analyzed under different information conditions. ERP II implementing options' decision-optimizing models were established. In these models, both clients and vendors try to pursue their own benefits. Based upon the principal-agent theory, the models show how a principal can force an agent to pursue his benefits. Finally, a simulation experiment regarding ERP II implementing options was made. The analysis result of ERP II implementing options is verified.
\end{abstract}

Index Terms -ERP II implementing problems; Asymmetry information; Implementing control cost; Evaluation level; Principal-agent; Solution

\section{INTRODUCTION}

There are large similarities between the way of handling ERP II implementation options and financial options, as will be demonstrated in this paper. Hence, extending financial options' theory models can help us deal with ERP II investment decisions [1]. The main idea is that vendors can buy ERP II implementation options that are offered by clients. That is, he can buy the right to implement. Clients can invest in the vendors' projects, and offer permission to implement. This allows the vendor to generate benefits in return for his investment. However, vendors may choose not to buy the rights, but this will lead to the risk of being forced to stop his ERP II project with because he has no effective implementing control. Because the cost of having to stop the project is must bigger than the cost of buying clients' ERP II implementation rights, a vendor commonly will choose buying ERP II implementation rights.

The dilemma of an ERP II (or ERP, this is more common) implementation is a bottleneck problem of disturbing industries' operation, esp. of supply chain (SC) integration benefits [2]. A client, core enterprise or other member enterprise in the SC adopts various measures to control the effect of an ERP II implementation and to make sure he gets a sufficient part of the gains. But, when serious asymmetry exists between the client and the ERP II vendor, an ERP II implementation becomes very difficult.

\author{
Koen Milis and Stephan Poelmans \\ Room 3113,EHSAL Research Center of HUB, Stormstraat 1, \\ 1000 Brussels, Belgium \\ Koen.milis@hubrussel.be \\ Stephan.poelmans@hubrussel.be
}

As principal, clients may have different characteristics; resulting in various requirements. At same time, the agent's (vendor) capabilities of implementing and maintaining clientspecific ERP II projects may be relatively low because he doesn't have all the information on the ERP II's real implementing effect on the client's operation processes. On the other hand, a vendor may only care about his standard ERP II implementation project, though this may not be suitable for the client's business. So, there is a possible trade-off between standardization and the capability to fulfill the needs of the clients. Moreover, clients do not necessarily have a big inside into the impact of an ERP II implementation since they might not be acquainted with the possibilities and limitations of an ERP II system. Often, they are not familiar with technological problems when facing an ERP II implementation, while the vendors are often not acquainted with the processes and operations of the enterprise or its supply chain. There is asymmetric information between client and agent.

In addition, according to asymmetry information theory, the vendors' private information on ERP II implementations is regarded as external, and thus can't be controlled. This is just regarded as a coincident to the hidden information concept, under the condition that the two players all can realize information asymmetry. This is inevitably reflected in their implementing controlling actions. In an environment of hiddeninformation, the major problem is, in the process of arranging options contract that the vendors' contract choices may conflict with what clients expect, and adverse choosing is formed. Under the condition of hidden-action, vendors also may start from his own benefit, and do some actions which damage the clients' benefit-then, moral risk is formed.

In the paper, ERP II implementing options' decisionoptimizing models were established. With clients as principal, vendors as agent, ERP II implementation evaluation and bad quality prevention principal-agent models were set up. In these models, both clients and vendors try to pursue their own benefits. Based upon the principal-agent theory, the models show how a principal can force an agent to pursue his benefits. 


\section{ERP II IMPLEMENTING-OPTIONS-DECISION'S PRINCIPAL AGENT MODEL}

As a principal, the clients' ERP II implementing benefit function, say, principal's target function is

$$
Z_{1}=P-C_{A}-\left(1-P_{A}\right) P_{E} W-\left(1-P_{A}\right)\left(1-P_{E}\right) X
$$

Here, $Z_{1}$ is the client-controlled implementation benefit.

$P$ is the vendors option payment which is asked by clients, that is, clients revenue -it is a decision variable, and is the function of the vendors implementation-evaluation level $P_{E}, P=P\left(P_{E}\right)$.

$C_{A}$ is the clients cost to prevent bad implementation quality, and is a function of $P_{A}, C_{A}=C_{A}\left(P_{A}\right)$.

$P_{A}$ is the bad quality implementation prevention level of clients, it describes implementation-controlling probability, so, it is a decision variable which is ascertained by clients and having subject active affection, $P_{A} \in[0,1], P_{A}=P_{A}\left(P_{E}\right)$.

$P_{E}$ is the implementation-evaluation level of the vendors, it describes the probability of vendor-controlled implementation that excesses standard. It is a decision variable which is ascertained by the clients and that has subject active affection,$P_{E} \in[0,1]$.

$W, X$ are certain constants that are negotiated by clients and vendors.

Hence, clients individual rational constraint ( $\left.I R_{C}\right)$ is $P_{A} \leq A$. Here, $A$ is the clients' highest implementingcontrolling level. It is her rational constraint of implementingprevent ability. For the convenience of dealing with problems, according to literature [11], change $I R_{C}$ to condition of twotimes model function, is

$$
Z_{2}=\frac{1}{2} a\left(P_{A}-A\right)^{2}
$$

Here, $a$ is parameter of vendors greatest implementingevaluation ability, and $a>0$.

Vendors implementing-controlling benefit function, that is, agent's target function is

$$
Z_{3}=P_{A} \pi_{G}+\left(1-P_{A}\right) P_{E}\left(U_{E}+W\right)+\left(1-P_{A}\right)\left(1-P_{E}\right)\left(\pi_{B}+X\right)-P-C_{E}
$$

Here, $Z_{3}$ is vendor's implementing-controlling benefit.

$\pi_{G}$ is vendors benefit when his implementing-releasing absolutely coincident to the standard.

$\pi_{B}$ is the benefit that vendors have potential situations of not coincident to standard, but he hasn't checked it out.

$U_{E}$ is prize which is given by clients when vendors have checked out the default.
$C_{E}$ is vendor implementation-evaluation cost, it is a function of $P_{E}, C_{E}=C_{E}\left(P_{E}\right)$.

In the problem of an ERP II implementing decision, clients must consider their benefit. So, $Z_{4}=b Z_{3}$ (here, $b$ is probability coefficient , $0 \leq b \leq 1$ ). Hence, according to (1), (2) and (3), clients benefit is

$$
Z=Z_{1}-Z_{2}+Z_{3}
$$

\section{ERP II IMPLEMENT ING-PREVENTING AND OPTIONS PAYMENT DECISIONS}

\section{A. ERP II implementation-preventing and Options Payment} Decisions under Symmetry Information

Under condition of s ymmetric information between clients and vendors, clients absolutely can observe vendors implementation-evaluation activities. Their ERP II implementation decision problem is an optimizing problem. Clients target is to choose appropriate implementationpreventing level $P_{A}$ and options payment $P$, and maximize implementation-controlling benefits, that is

$$
\max Z=\underset{P_{A}, P}{Z}\left(P_{A}, P\right)
$$

At the same time, under principal-agent theory, as a principal, clients have rights to make the vendors benefit zero, say, $Z_{3}=0$, then

$$
P=P_{A} \pi_{G}+\left(1-P_{A}\right) P_{E}\left(U_{E}+W\right)+\left(1-P_{A}\right)\left(1-P_{E}\right)\left(\pi_{B}+X\right)-C_{E}
$$

Take (6) into (4), and seek one time derivative of $P_{A}$, and make it zero, through arranging, we get

$$
C_{A}^{\prime}+a\left(P_{A}-A\right)=P_{E} W+\left(1-P_{E}\right) X
$$

Second derivative of (4) is $\frac{d^{2} Z}{d P_{A}^{2}}=-C_{A}^{\prime}-a<0$, (5) shows the maximum. Vendors implementation-evaluation and options payment decision are

$$
\begin{aligned}
& P_{A}=P_{A}\left(P_{E}\right) \\
& P=P\left(P_{E}\right)
\end{aligned}
$$

\section{B. ERP II Implementing-Preventing and Options Payment Decisions under Asymmetry Information}

Now, consider the situation were vendors have ERP II implementation knowledge (private information), and clients can't observe this. The ERP II implementing decision problem becomes an asymmetric information problem, which is a typical principal-agent problem [3]. In the condition of agent-vendors' ERP II implementing evaluation information hidden, clients target function can be deszcribed as shown in formula 10. The 
Clients will choose certain implementing-prevention level $\mathrm{P}_{\mathrm{A}}$ and options payment $P$ to make target function (4) maximumunder condition of expectation, that is

$$
\max _{P_{A}\left(P_{E}\right), P} E(Z)=\int_{P_{E}^{L}}^{P_{E}^{H}} Z f\left(P_{E}\right) d P_{E}
$$

Here, $E(Z)$ is the clients target benefit function under asymmetric information. $P \in\left[P_{E}^{L}, P_{E}^{H}\right]$, and obeys probability distribution with probability density $f\left(P_{E}\right)$. And now, clients can estimate the vendor's implementingevaluation level, say, to clients, vendors implementingevaluation level $P_{E}$ is an estimated value $\hat{P}_{E}$. This will ask clients to design a stimulus plan to proclaim $\hat{P}_{E}$ that is close to the real implementing-evaluation level, and guides vendors to get certain target. At the same time, clients can also realize his target $[4,5]$.

According to the proclaim axiom of principal-agent theory $[6,7]$, there is $\hat{P}_{E}=\underset{\hat{P}_{E}}{\arg \max } Z_{3}\left(\hat{P}_{E}\right)=\underset{\hat{P}_{E}}{\arg \max }\left[P_{A}\left(\hat{P}_{E}\right) \pi_{G}+\left(1-P_{A}\left(\hat{P}_{E}\right)\right) P_{E}\left(U_{E}+W\right)+\left(1-P_{A}\left(\hat{P}_{E}\right)\right)\left(1-P_{B}\right)\left(\pi_{B}+X\right)-P\left(\hat{P}_{E}\right)-C_{E}\right]$

Clients model's condition (11) can change to a one time condition, ask for a first degree derivative of $\hat{P}_{E}$ and make it zero, that is

$$
\begin{aligned}
& \frac{d P}{d \hat{P}_{E}}=\left[\pi_{G}-P_{E}\left(U_{E}+W\right)-\left(1-P_{E}\right)\left(\pi_{B}+X\right)\right] u \\
& \quad \frac{d P_{A}}{d \hat{P}_{E}}=u
\end{aligned}
$$

Here, $u$ is the first degree derivative of implementingprevention level $\boldsymbol{P}_{A}$ to implementing-evaluation level $\boldsymbol{P}_{E}$ 's estimated value $\hat{P}_{E}$, and is an introduced controlling variable.

Hence, the ERP II bad implementation quality prevention problem under asymmetric information can be regard as optimum control problem that has target function: benefit expectation (10) and status (12) and (13). In the optimum control problem, all implementing-evaluation level variables are all become to $P_{E}$. Because through proclaiming an axiom and stimulus strategy, the vendors implementation-evaluation level comes closed to the real value. So, in the next steps real implementing-evaluation level $\boldsymbol{P}_{E}$ is applied.

Using Maximum axiom to seek a solution for a classic controlling problem-through (10), (12) and (13) to establish Hamilton function as follows

$H=Z f\left(P_{B}\right)+\lambda_{P}\left[\pi_{G}-P_{E}\left(U_{E}+W\right)-\left(1-P_{E}\right)\left(\pi_{B}+X\right)\right] u+\lambda_{P_{A}} u$

Here, $\lambda_{P}, \lambda_{P_{A}}$ are joint variables of problems.

Controlling equation is

$\frac{\partial H}{\partial u}=\lambda_{P}\left[\pi_{G}-P_{E}\left(U_{E}+W\right)-\left(1-P_{E}\right)\left(\pi_{B}+X\right)\right]+\lambda_{P_{A}}=0$

Joint equations are

$$
-\frac{d \lambda_{P}}{d P_{E}}=\frac{\partial H}{\partial P}=(1-b) f\left(P_{E}\right)
$$

$-\frac{d \lambda_{P}}{d P_{E}}=\frac{\partial H}{\partial P_{A}}\left\{-C_{A}+P_{E} W+\left(1-P_{E}\right) X-a\left(P_{A}-A\right)+b\left[\pi_{G}-P_{E}\left(U_{E}+W\right)-\left(1-P_{E}\right)\left(\pi_{E}+X\right)\right]\right\} f\left(P_{E}\right)$

From (16), get

$$
\lambda_{P}=(b-1) F\left(P_{E}\right)
$$

Here, $F\left(P_{E}\right)$ is probability distribution function of implementing-evaluation parameter $P_{E}$.

Unite (15), (17) and (18), get clients implementingprevention decision solution under asymmetric information is

$$
P_{A}^{V}=P_{A}\left(a, b, P_{E}, f, F\right)
$$

So, the solution of the options payment is $\frac{d P_{A}^{V}}{d P_{E}}=\left.\left[\pi_{G}-P_{E}\left(U_{E}+W\right)-\left(1-P_{E}\right)\left(\pi_{B}+X\right)\right] \frac{d P_{A}}{d P_{E}}\right|_{P_{A}=P_{A}^{V}}$.

\section{ANALYSES OF DECISION RESULTS}

Consider the clients implementing-prevention cost function $C_{A}$ to implementing-prevention level $P_{A}$ 's first and second degree derivatives, which are all greater than 0 , and for the convenience of dealing, choose literature [8]'s prevention cost function $C_{A}\left(P_{A}\right)=\frac{1}{2} K_{A} P_{A}^{2}$. Here, $K_{A}$ is the coefficient to be decided upon. From (8) and (19), get

$$
\begin{gathered}
\boldsymbol{P}_{A}=\frac{\boldsymbol{a} \boldsymbol{A}+\boldsymbol{P}_{E} \boldsymbol{W}+\left(\mathbf{1}-\boldsymbol{P}_{\boldsymbol{E}}\right) \boldsymbol{X}}{\boldsymbol{K}_{\boldsymbol{A}}+\boldsymbol{a}} \\
P_{A}^{V}=\frac{a A+P_{E} W+\left(1-P_{E}\right) X}{K_{A}+a}+\frac{\pi_{G}-P_{E}\left(U_{E}+W\right)-\left(1-P_{E}\right)\left(\pi_{B}+X\right)}{K_{A}+a}+\frac{(1-b) F\left(\pi_{E}-U_{E}-W+X\right)}{\left(K_{A}+a\right) f}
\end{gathered}
$$

Compare implementing-evaluation decision (20) under symmetric information with implementing-evaluation decision (21) under asymmetric information, we discovered that there is an increment, it is

$$
\Delta P_{A}=\frac{\pi_{G}-P_{E}\left(U_{E}+W\right)-\left(1-P_{E}\right)\left(\pi_{B}+X\right)}{K_{A}+a}+\frac{(1-b) F\left(\pi_{E}-U_{E}-W+X\right)}{\left(K_{A}+a\right) f}
$$

Now, we will analyze implementing-prevention decision results under different information conditions. There is $\pi_{B}-U_{E}=\left(\pi_{G}-U_{E}\right)-\left(\pi_{G}-\pi_{B}\right)$. Here, $\left(\pi_{G}-U_{E}\right)$ is the vendors inter loss cost and $\left(\pi_{G}-\pi_{B}\right)$ is the vendors outer loss cost. Suppose that vendors inter loss implementation punishment is at-least equal to inter loss, $W \geq \pi_{G}-U_{E}$, and suppose that vendors outer loss implementing punishment is at least equal to outer loss, $X \geq \pi_{G}-\pi_{B}$. Here consider

$$
\begin{gathered}
\pi_{G}-U_{E}=\frac{W}{N} \\
\pi_{G}-\pi_{B}=\frac{X}{N}
\end{gathered}
$$

Here, $N$ is constant greater or equal to 1 .

Take (23), (24) into (22), get 


$$
\Delta P_{A}=\frac{N-1}{\left(K_{A}+a\right)}\left[\frac{(1-b) F}{f}(X-W)-P_{E} W-\left(1-P_{E}\right) X\right]
$$

From (25), we can know, when $W>X, W-X>0$, $\Delta P_{A}<0$, now, $P_{A}^{V}<P_{A}$. That is, when vendors inter loss punishment from clients is greater than outer loss punishment, clients' implementing-prevention level under asymmetric information is less than that under symmetric information. When $N=1, \Delta P_{A}=0$, now, $P_{A}^{V}=P_{A}$, say, when vendors inter loss punishment from clients is equal to inter loss cost and his outer loss punishment equal to outer loss cost, clients implementing-prevention level under asymmetric information is equal to the results under symmetric information.

When $W<X$, along $X$ far greater than $W, X-W \approx X, \Delta P_{A}=\frac{N-1}{\left(K_{A}+a\right) N}\left[\frac{(1-b) F}{f}\left(1-P_{E}\right)\right] X$. Suppose implementing-evaluation parameter $P_{E} \in[0.8,0.9]$, it obeys to uniform distribution $f\left(P_{E}\right)=10$, get $0.1 \leq 1-P_{E} \leq 0.2$. And still, $0 \leq b \leq 1$, so, $0 \leq 0.1(1-b) \leq 0.1$, $0.1(1-b)-\left(1-P_{E}\right) \leq 0$, hence, ${ }_{A} P_{A}=\frac{N-1}{N}\left[0.1(1-b)-\left(1-P_{E}\right)\right] X \leq 0$, now, $P_{A}^{V} \leq P_{A}$, that is, when vendors inter loss punishment from clients is less than outer loss punishment, even when his outer loss punishment far exceeds the inter loss punishment, the clients implementing-prevention level under asymmetric information will not be higher than the result under symmetric information.

In a word, whether the vendors inter loss punishment from clients is greater or smaller than the outer loss punishment, clients' implementing-prevention level decision results under asymmetric information are all lower than under symmetric information. That is because, under asymmetric information, clients cannot observe vendors implementingevaluation activities, the client has to do his best to lower implementing cost-that is, implementing prevention cost-from a client's point of view. This is direct result from lowering implementing prevention level.

When clients make implementation prevention level decisions, the overall implementation-control cost triggered includes implementation prevention and implementation punishment cost. The mathematic equation is

$$
C=\left(1-P_{A}\right) P_{E} W+\left(1-P_{A}\right)\left(1-P_{E}\right) X+C_{A}
$$

When $a=0$, take (20) under symmetric information and (21) under as ymmetric information into (26), get

$$
C_{P_{A}^{\nu}}-C_{P_{A}}=\frac{(N-1)^{2}}{2 K_{A} N^{2}}\left[\frac{(1-b) F}{f}(X-W)-P_{E} W-\left(1-P_{E}\right) X\right]^{2}
$$

Here, $C_{P_{A}^{V}}$ is client implementation-control cost under asymmetric information. $C_{P_{A}}$ is the client's implementationcontrol cost under symmetric information. Equation (27) shows, clients implementing-controlling cost under asymmetric information is higher or equal to the result under symmetric information. When $N=1, C_{P_{A}^{V}}-C_{P_{A}}=0$. Say, when the vendor's inter loss punishment is equal to inter loss cost and his outer loss punishment equal to outer loss cost, clients implementation-control cost under asymmetric information is higher than the result under symmetric information. This shows that, under asymmetric, as principals, the strategies of stimulating vendors, must pay implementation-control cost.

\section{SIMULATION CALCULATIONS}

Here, an implementation prevention decision problem of a client to a vendor under asymmetric information is discussed. The client's achieved benefit is $\pi_{G}=5000$ EUR, when vendor is absolutely coincident to the standard. The client checked out the implementation, and vendor timely adopted implementation-control measures, the client's benefit is $U_{E}=4000$ EUR. The client achieved market benefit is $\pi_{B}=3000 \mathrm{EUR}$, when the vendor has the possibility of potential excess standard. The client punishes the vendor inter loss $W=2000$ EUR; the outer loss punishment of the vendor is $X=4000$ EUR.

The client's highest implementing prevention level is $A=0.98$. In addition, $a=0.1, b=0.1$. Consider the vendor's implementing evaluation cost function $C_{E}\left(P_{E}\right)$ to implementing evaluation level $P_{E}$ first and second degree derivatives all greater than 0 , the client's implementation prevention cost function $C_{A}\left(P_{A}\right)$ to achieve implementation prevention level $P_{A}$ first and second degree derivatives all greater than 0 , and for the convenience of dealing, choose vendor's evaluation cost function $C_{E}\left(P_{E}\right)=\frac{1}{2} K_{E} P_{E}^{2}$, the client's implementing prevention cost function $C_{A}\left(P_{A}\right)=\frac{1}{2} K_{A} P_{A}^{2}, K_{A}=K_{E}=4000$. Suppose that vendor's implementing evaluation coefficient $P_{E} \in[0.8,0.9]$ and it obeys uniform distribution $f\left(P_{E}\right)=10 \cdot P_{E}$ has the value $P_{E}=P_{B_{0}}+n \Delta P_{E}=0.8+0.005 n, n=1,2, \cdots, 20$. The decision results are as Fig. 1 and Fig.2. Here, full line is decision results under symmetric information, whereas imaginary line is the results under asymmetric information.

Fig.1 shows, the client's implementation prevention level under asymmetric information is lower than that under symmetric information. Fig.2 shows, the client's implementation-control cost under asymmetric information is higher than that under symmetric information, the asymmetric information make the client paying the price of implementing controlling cost. 


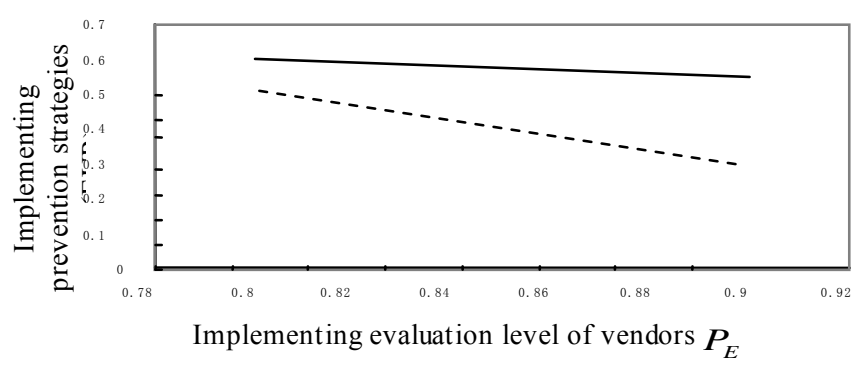

Fig. 1 Implementing prevention strategies under different information

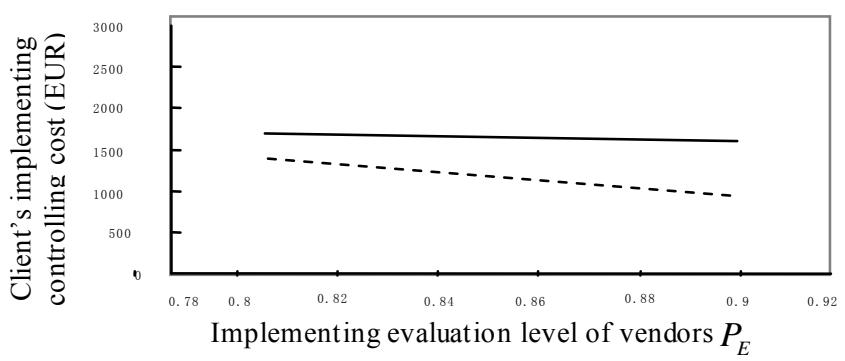

Fig. 2 Client's implementing controlling cost under different information

\section{CONCLUSIONS}

1. In the paper, option theory and the theory of asymmetric information were used to examine ERP II implementation decisions under symmetrical and asymmetrical information situations. ERP II implementation options' decision-optimizing models were established. ERP II implementation prevention and evaluation principal-agent models were set up. In these models, both clients and vendors try to maximize their own benefits.

2. Under condition of asymmetric information, vendors hide there implementing evaluation information. Using maximum axiom deduced the solution projects of ERP II implementing options optimum investing and options payment. The results show, could use theories of options and asymmetric to solve ERP II implementing options decision.

3. Also, simulation figure show, the client's implementation prevention level under asymmetric information is lower than that under symmetric information. In addition, the client's implementationcontrol cost under asymmetric information is higher than that under symmetric information, the asymmetric information make the client paying the price of implementing-controlling cost.

\section{REFERENCES}

[1] Corbett C J, Groote X D, “A supplier's optimal quantity discount policy under asymmetric information", Management Science, 2000, 46(3), pp. 445-450.

[2] Cobett C J, Tang C S, "Designing supply contracts: Contract type and information asymmetry", in: Tayur S, Ganeshan R, Michael M. ed, Quantitative Models for Supply Chain Management. Kluwer Academic Publishers, pp. 269-298, 2000.

[3] Gauder G, Pierre L, Long I V, "Real investment decision under adjustment costs and asymmetric information", Journal of Economic Dynamics and Control, 1998, 23(1), pp. 71-95.

[4] H. X. Yuan, Z. X. Tian, "Material options optimum investment problem research under asymmetric information", Management Science Journal, 2003, 6(6), pp. 28-33 (in Chinese).

[5] Jim Ayers; "Is Supply Chain Management the Same as ERP"; Project Management; spring 2001, p 43-45

[6] Kleijnen J. \& Gaury E.; "Robustness in simulation: a practical methodology"; Eur. J. opl. Res, accepted for publication.

[7] T say A, Nahmias S, "Modeling supply chain contracts: A review", in: Tayur S, Ganeshan R, Michael M. ed, Quantitative Models for Supply Chain Management, Boston: Kluwer Academic Publishers, pp. 299-336, 2000.

[8] Yeom S, Blachandran K, Ronen J, "The role of transfer price for coordination and control within a firm", Review of Quantitative Finance and Accounting, 2000, 14(2), pp. 161-192. 